\title{
Precision of Interferometric Diffusion Coefficients in a Four-Component System Relevant to Protein Crystal Growth: Lysozyme-Tetra(ethylene glycol) $-\mathrm{NaCl}-\mathrm{H}_{2} \mathrm{O}$
}

\author{
Onofrio Annunziata, ${ }^{\dagger}$ Alessandro Vergara, ${ }^{\ddagger}$ Luigi Paduano, ${ }^{\ddagger}$ Roberto Sartorio, ${ }^{*, \ddagger}$ \\ Donald G. Miller, ${ }^{\S}$ and John G. Albright ${ }^{\#}$ \\ Massachusetts Institute of Technology, Cambridge, Massachusetts, Chemistry Department, University \\ "Federico II" of Naples, via Cinthia 80126 Naples, Italy, Lawrence Livermore National Laboratory, \\ Livermore L-203, California 94550, and Texas Christian University, Fort Worth, Texas 76129
}

Received: January 27, 2003; In Final Form: March 31, 2003

\begin{abstract}
We investigate the precision of Rayleigh interferometry in evaluating the nine mutual diffusion coefficients $D_{i j}$ defining a four-component system by investigating the system lysozyme-tetra(ethylene glycol) $-\mathrm{NaCl}-$ $\mathrm{H}_{2} \mathrm{O}$. We believe our results to be the most successful experimental determination of diffusion coefficients in quaternary systems. This choice of system is motivated by its relevance to protein crystal growth. The comparison of the $D_{i j}$ coefficients of the quaternary system and the $D_{i j}$ coefficients from the corresponding ternary and binary systems enables us to extract some information about the hydrodynamics and thermodynamics in solutions where protein crystals grow.
\end{abstract}

\section{Introduction}

Optical interferometry provides the most precise methods for determining mutual diffusion coefficients in liquids.

The principal purpose of this methodological paper is to test the precision of diffusion coefficient measurements by Rayleigh interferometry in a four-component system, relevant to crystal growth. The motivation follows.

The quality of protein crystals is important for determining their X-ray structures. On the surface, diffusion, convection, and sedimentation are significant transport processes that are involved in the nucleation and growth of protein crystals; however, convection and sedimentation are detrimental. In microgravity ${ }^{1}$ and gelled media, ${ }^{2}$ the protein transport is essentially diffusive. In either case, diffusion data are particularly important for modeling the various growth processes.

Solutions used to precipitate proteins typically contain one or more other solutes, such as salts, organic solvents, and polymers. A single additional component gives a threecomponent system; two additional components give a fourcomponent system. It has been customary in protein solution studies to describe these diffusing systems by a single pseudobinary diffusion coefficient. However, the need for a reference frame (here, the volume-fixed frame) reduces the number of independent fluxes to $n-1$. Therefore, an $n$-component diffusing system has $(n-1)^{2}$ diffusion coefficients $D_{i j}$, which comprise the diffusion tensor: 4 for a three-component system, 9 for a four-component system. We have found experimentally that a cross term $D_{i j}(i \neq j)$ can be very large in three-component aqueous systems containing lysozyme and a single salt, and this cross term is significantly larger than both main terms $D_{i i}{ }^{3-5}$ Consequently, the pseudo-binary approximation is quite inadequate for modeling diffusive transport in crystal growth.

* Author to whom correspondence should be addressed. E-mail: Sartorio @ chemistry.unina.it. Fax: 39081674090.

†assachusetts Institute of Technology.

‡ University "Federico II" of Naples.

$\S$ Lawrence Livermore National Laboratory.

* Texas Christian University.
In this protein context, we find that the flows of the components calculated assuming cross-term diffusion coefficients are zero (i.e., setting $D_{i j}=0, i \neq j$ ) can differ by as much as $10 \%-15 \%$ from flow values calculated with actual nonzero cross terms. These differences can have a profound effect. Neglecting cross terms can lead to the erroneous conclusion that nonprotein solute concentrations are almost the same throughout the solution, including up to the crystal interface. However, including the cross-term diffusion coefficients $D_{i j}$ in model calculations shows that there can be a variation of several percent in the nonprotein solute concentration at the crystal surface, which can change as a function of time. Such variation with time can increase precipitant concentration and affect nucleation. It can also influence water and impurity inclusion in the crystal as it grows, which can cause defects, degrading the quality of the crystal.

One technique for nucleation and crystal growth in microgravity is the free interface diffusion method, where a proteinrich solution and a precipitant-rich solution diffuse together to produce nucleation conditions and then further diffuse toward a uniform concentration distribution having slow growth conditions. Because nucleation conditions are sensitive to small concentration differences, cross-term diffusion coefficients are also important here.

For the previously described reasons, experimental diffusion studies in multicomponent protein systems have generated considerable interest. Our previous studies involved the threecomponent systems that had aqueous lysozyme (a representative protein) and a single additional component.

The group in Fort Worth, TX, has focused on threecomponent aqueous lysozyme systems with single-salt precipitants and has been supported by the National Aeronautics and Space Administration (NASA). ${ }^{3-5}$ The group in Naples, Italy has focused on three-component aqueous lysozyme systems with polymer additives, such as poly(ethylene glycol) (PEG) of varying molecular weights, and has been supported by ASI (the Italian Space Agency). ${ }^{6,7}$ Both groups have collaborated closely for many years. Because most protein precipitation protocols 
involve at least four components, it seems desirable to examine the precision of diffusion measurements for a four-component lysozyme system that contains a salt plus a PEG polymer.

Unfortunately, most polymer additives, such as PEG, are polydisperse, which already makes them multicomponent systems by themselves and, thus, considerably complicates the data analysis. ${ }^{8,9}$ It is possible to partially correct for polydispersity; however, accuracy is reduced. ${ }^{10}$ Therefore, we have chosen monodisperse PEG4 as the polymer additive as a way of checking our ability to make precision measurements of the $D_{i j}$ of a four-component system. The test system chosen is lysozyme (component 1, $0.6 \mathrm{mM}$ )-PEG4 (component 2, 0.5 $\mathrm{M})-\mathrm{NaCl}$ (component $3,0.5 \mathrm{M}$ ) $-\mathrm{H}_{2} \mathrm{O}$ (component 0 ) at $\mathrm{pH}$ 4.5 and $298.15 \mathrm{~K}$. It represents a good example of a fourcomponent protein-precipitant system, even though PEG4 itself is not usually used as a precipitant.

There are few published results of the nine $D_{i j}$ diffusion coefficients for four-component systems. These include measurements by diaphragm cells ${ }^{11-13}$ and Taylor dispersion, ${ }^{14-16}$ one of which involved protein systems. ${ }^{16}$ The only previous interferometric results ${ }^{17}$ were obtained at the Naples facility by Gouy interferometry on the system cyclodextrin-phenyalaninemonobutylurea $-\mathrm{H}_{2} \mathrm{O}$.

The present work was done in collaboration at Texas Christian University in Forth Worth using automated Rayleigh interferometry on the Gosting diffusiometer, ${ }^{18}$ which is the world's highest precision apparatus for optical diffusion measurements. Gouy and Rayleigh measurements should give the same results in principle and are extremely similar in practice. ${ }^{19-21}$ However, Rayleigh interferometry is the easiest for automation, which makes many more scans possible, which, in turn, give higher precision. Furthermore, in contrast to Gouy, extraction of the $D_{i j}$ from the Rayleigh fringe positions is more direct, and very dilute systems with few fringes can be analyzed more precisely.

We will find that the four-component Rayleigh data from the automated Gosting diffusiometer have considerably better precision than the data from the earlier four-component Naples measurements, with most $D_{i j}$ errors being quite small. We will also see that some cross-term coefficients are large, and that $D_{31}$ is 7 times larger than the largest of the main terms.

We will also provide some comparisons of the interesting specific effects of adding PEG4 to the ternary protein system lysozyme $-\mathrm{NaCl}-\mathrm{H}_{2} \mathrm{O}$.

\section{Data Analysis}

Kim considered various possible analysis methods for obtaining the nine $D_{i j}$ coefficients from four-component Gouy fringe pattern data in 1966 and 1969,,$^{22,23}$ one of which involved combining Rayleigh and Gouy fringe patterns. However, no actual working procedure was presented.

In 1972, Eppstein and Miller, following a suggestion by Albright, worked out the first successful analysis of Rayleigh fringes from ternary systems. It was described briefly in various papers, and in detail in ref 19. In 1973, one of us (DGM) also realized that the principles of the ternary analysis could be directly extended to four-component systems. At that time, he performed the complex algebra that related the nine $D_{i j}$ coefficients to the nine parameters determined by nonlinear leastsquares calculations from the Rayleigh fringe position measurements.

High precision in the four-component fringe positions is essential, because the uncertainties from least-squares calcula- tions for the nine parameters of a four-component system will be larger than those for the four parameters of a three-component system. Such precision was not available to us in 1973. As a result, the four-component Rayleigh analysis procedure was put aside.

The Gosting diffusiometer ${ }^{18}$ became available to us in 1981 . In 1987, it was discovered that an analysis of Gouy data could be put into a form directly analogous to the Rayleigh analysis, with the same least-squares parameters and same equations relating them to the $D_{i j}$ coefficients. These Gouy results and the earlier Rayleigh analysis were published in 1988 for three- and four-component systems with distinct eigenvalues. $^{24}$ An analysis of equal eigenvalues in three- and fourcomponent Rayleigh systems has also been presented. ${ }^{25}$ The new Gouy procedure was applied to the four-component data from the diffusiometer at the Naples facility, as noted previously. ${ }^{17}$

Subsequently, the four-component Rayleigh analysis was incorporated into the automated procedure for the Gosting machine measurements. With automation, the Gosting machine's already high precision has been improved enough to get excellent values of the nine four-component $D_{i j}$ coefficients.

The general outline of the data analysis is given in ref 24, and the relevant equations for free-diffusion boundary conditions are

$$
\begin{gathered}
f(j)=\frac{2 j-J}{J}=\Gamma_{1} \operatorname{erf}\left(s_{1} y_{j}\right)+\Gamma_{2} \operatorname{erf}\left(s_{2} y_{j}\right)+\Gamma_{1} \operatorname{erf}\left(s_{3} y_{j}\right) \\
\Gamma_{1}=a_{1} \alpha_{1}+a_{2} \alpha_{2}+a_{3} \alpha_{3} \\
\Gamma_{2}=b_{1} \alpha_{1}+b_{2} \alpha_{2}+b_{3} \alpha_{3} \\
\Gamma_{3}=1-\Gamma_{1}-\Gamma_{2}
\end{gathered}
$$

where $j$ is a number defining the sequence number of a fringe center in the Rayleigh pattern at a position $y_{j} ; y_{j}$, which is defined below, is the reduced fringe position corresponding to $j ; J$ is the total number of interference fringes (generally not an integer); and the $s_{i}$ parameters are $1 / \sqrt{\lambda_{i}}$, where $\lambda_{i}$ are the eigenvalues of the diffusion coefficient matrix. Earlier nonautomated analysis methods used the fringe position corresponding to an integer value of $j$. Our current method uses fixed positions and associates a value $j$ (now the center of a shifted fringe) to each position; these $j$ values are not necessarily integers.

The $\alpha_{i}$ parameters are the refractive index fractions:

$$
\alpha_{i}=\frac{R_{i} \Delta c_{i}}{\sum_{k=1}^{3} R_{k} \Delta c_{k}}
$$

The $R_{i}$ parameters are the refractive index increments that best relate the total number of fringes $J$ to the concentration differences $\Delta c_{i}$ across the initial boundary for a given experiment according to the equation

$$
J=\sum_{k=1}^{3} R_{k} \Delta c_{k}
$$

A minimum of three experiments is required with different $\Delta c_{i} / \Delta c_{j}$ ratios to isolate the three $R_{i}$ values. The values of the three $R_{i}$ parameters were determined by the method of least squares by weighting the sets of $J$ and $\Delta c_{i}$ values from all the experiments equally. 
The $y_{j}$ values are the reduced fringe positions:

$$
y_{j}=\frac{x_{j}-x_{J-j}}{4 \sqrt{t}}
$$

where $x_{j}$ and $x_{J-j}$ are the positions of the symmetrically paired fringes $j$ and $(J-j)$ (Creeth pairs) ${ }^{26}$ and $t$ is the corrected time of starting the run. ${ }^{27}$ Each experiment has $\sim 50$ scans at different "clock" times, which permits this correction of the experimental clock time by a value $\Delta t$, the time from an infinitely sharp boundary to the start of the clock time. Symmetrical pairing of the fringes eliminates both a concentration dependence and a large optical aberration (Wiener skewness). ${ }^{26}$ For each $j$ in a given experiment, the values of $y_{j}$ after time correction should be the same for each scan, so they can be averaged in preparation for the overall least-squares calculation.

The $f(j)$ and symmetrically paired fringes are obtained as follows.

The automation procedure collects several intensities for each pixel in each horizontal scan of the 6000 vertically arranged pixels. The minimum intensity in the horizontal direction is determined for each pixel by least-squares calculation. Pixels in the top part of the fringe pattern (after baseline correction ${ }^{19}$ ) have a fixed horizontal minimum position, because the fringe is vertical there. This position is denoted as fringe " 0 ". As the boundary is entered when one moves downward (i.e., with increasing $x_{j}$ and $y_{j}$ ), the corresponding pixels show a shift of horizontal position of the minimum. This shift, divided by the interfringe distance, is a fringe shift, $j$. Moving further downward into the boundary, the minimum intensity position approaches the "edge" of the diffraction pattern. At this point, the minimum intensity position that is one interfringe distance back is now taken as the position shift for this pixel, but a value of 1 is added to the corresponding fringe shift. This process is repeated until the downward recording of minima is outside the boundary and into the vertical fringe in the bottom part of the pattern. The final total fringe shift is the total number of fringes, $J$, and the agreement of its value with that obtained by the fractionalpart-of-the-fringe measurement ${ }^{19}$ is a useful diagnostic.

At this point, there is a value of $j$ for each pixel of the 6000 pixels. From them, the reduced fringe number, $f(j)=(2 j-J) /$ $J$, of eq 1 is obtained for each pixel of that scan. The values of $j$ run from 0 to $J$, and those for $f(j)$ run from -1 to +1 .

Within the diffusion boundary portion of the fringe pattern, the vertical positions for a set of 100 evenly spaced $f(j)$ values between $j=0$ and $j=J$ are obtained for each scan by curvefitting procedures. These positions and the mean time of the scan are stored for each scan. Initial solute concentrations for the experiment are also stored. These data, from three or more experiments, are recalled by a program (QFIT) and used to calculate the nine quaternary diffusion coefficients and give an estimate of their uncertainty. Separations of symmetric $f(j)$ values are first calculated by the QFIT program, ${ }^{27}$ and these are then used to calculate the quaternary diffusion coefficients.

A four-component system requires a minimum of three experiments, which must have different $\alpha_{i}$ values, or else the parameters $a_{i}$ and $b_{i}$ cannot be distinguished. Typically, seven experiments are used to improve statistics and check for any bad experiments. The seven sets of $\alpha_{i}\left(\alpha_{1}, \alpha_{2}, \alpha_{3}\right)$ are best chosen to be as orthogonal as possible. These correspond to $\mathrm{A}(1,0,0)$, $\mathrm{B}(0,1,0), \mathrm{C}(0,0,1), \mathrm{D}(1 / 2,1 / 2,0), \mathrm{E}\left({ }^{1} /{ }_{2}, 0,1 / 2\right), \mathrm{F}(0,1 / 2,1 / 2)$, and $\mathrm{G}(1 / 3,1 / 3,1 / 3)$.

The experimental quantities for each of the experiments are $j, J, x_{j}, t$, and, therefore, $f(j)$ and $y_{j}$. The nine experimental least- squares parameters are the three $a_{i}$ values, the three $b_{i}$ values, and the three $s_{i}$ values of eqs $1-3$. They are obtained by nonlinear least-squares calculations from the seven sets of $f(j)$ data from the seven corresponding experiments, using the Marquardt method. ${ }^{27}$ This iterative procedure requires initial values of the parameters $a_{i}, b_{i}$, and $s_{i}$. Suitable values, based on the assumption that $D_{i j}=0$ (for $i \neq j$ ), are $\left(a_{1}=1, a_{2}=a_{3}=\right.$ $0),\left(b_{2}=1, b_{1}=b_{3}=0\right)$, and $\left(s_{1}=1 / \sqrt{D_{1}}, s_{2}=1 / \sqrt{D_{2}}, s_{3}=\right.$ $\left.1 / \sqrt{D_{3}}\right)$. The values of $D_{i}$ are obtained by treating each of the three runs with $\alpha_{i}=1$ as a binary.

The nonlinear least-squares procedure now follows steps 4-7 in the Analysis of Data section of ref 17.

\section{Experimental Section}

1. Materials. Hen egg-white lysozyme, recrystallized six times and lyophilized, was purchased from Seikagaku America and used without further purification. The molecular mass of lysozyme solute was taken to be $14307 \mathrm{~g} \mathrm{~mol}^{-1}$, and this value was used to calculate all molar concentrations, after correction for the moisture and chloride content. ${ }^{3,4}$ Tetra(ethylene glycol) (PEG4) was purchased from Aldrich (purity listed as 99\%+) and used without further purification. The molecular mass of PEG4 solute was taken to be $194.23 \mathrm{~g} \mathrm{~mol}^{-1}$, and this value was used to calculate all molar concentrations. $\mathrm{NaCl}$ was purchased from Aldrich (purity listed as $99.9 \%$ ), dried by heating at $450{ }^{\circ} \mathrm{C}$ (as recommended by $\operatorname{Rard}^{28}$ ), and used without further purification. Its molar mass was taken to be $58.443 \mathrm{~g} \mathrm{~mol}^{-1}$, and its crystal density was taken to be $2.165 \mathrm{~g} \mathrm{~cm}^{-3}$, for buoyancy corrections. ${ }^{29}$ Deionized water was distilled and then passed through a four-stage Millipore filter system to provide high-purity water for all the experiments. The molar mass of water was taken to be $18.015 \mathrm{~g} \mathrm{~mol}^{-1}$.

2. Preparation of Solutions. All solutions were prepared by weight, using a Mettler Toledo model AT400 electro balance. The quaternary solutions used for diffusion and viscosity experiments were prepared using dry $\mathrm{NaCl}$ and stock solutions for both lysozyme-water and PEG4-water. A detailed description of the preparation of solutions has been described elsewhere. . $^{3,4}$

3. Density Measurements. All density measurements were performed with a Mettler-Paar model DMA40 density meter that was thermostated with water from a large, well-regulated $\left( \pm 0.01{ }^{\circ} \mathrm{C}\right)$ water bath. This instrument is interfaced to a computer for time averaging and gives a precision of $\pm 2 \times$ $10^{-5} \mathrm{~g} \mathrm{~cm}^{-3}$ or better. ${ }^{3,4}$

4. Viscosity Measurements. Viscosity measurements were performed on both the binary system PEG4 $-\mathrm{H}_{2} \mathrm{O}$ and the ternary system $\mathrm{PEG} 4-\mathrm{NaCl}-\mathrm{H}_{2} \mathrm{O}$, using an Ubbelhode viscometer in a bath at $25.00 \pm 0.01{ }^{\circ} \mathrm{C}$, with the viscosity of water as a reference. ${ }^{30}$

5. Free-Diffusion Measurements. The Gosting diffusiometer, used for our diffusion experiments, was designed and assembled at the Institute for Enzyme Research in Madison, WI, during the 1960s and early 1970s under the direction of Louis J. Gosting. ${ }^{18}$ It was subsequently at the University of Connecticut, then at Lawrence Livermore National Laboratory, and is now at Texas Christian University. It uses the Philpot-Cook arrangement of Rayleigh optics. ${ }^{31}$

The light source was a $\mathrm{He}-\mathrm{Ne}$ green laser, with a wavelength of $543.5 \mathrm{~nm}$ in air. The automation of fringe pattern acquisition in real time with a scanner using a $6 \mathrm{~cm}$ photodiode array, which was designed and constructed by M. E. Zeidler, is described by Rard et al. ${ }^{27}$ The principles of the Rayleigh procedure, although in terms of reading photographic plates, are detailed 
TABLE 1: Individual Experiments

\begin{tabular}{|c|c|c|c|c|c|c|c|}
\hline & $\begin{array}{c}\mathrm{A} \\
\alpha=[1,0,0]\end{array}$ & $\begin{array}{c}\text { B } \\
\alpha=[0,1,0]\end{array}$ & $\begin{array}{c}\mathrm{C} \\
\alpha=[0,0,1]\end{array}$ & 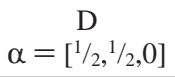 & $\alpha=\left[\begin{array}{l}E \\
\alpha / 2,0,1 / 2]\end{array}\right.$ & $\alpha=[0,1 / 2,1 / 2]$ & $\begin{array}{c}\mathrm{G} \\
\alpha=[1 / 3,1 / 3,1 / 3]\end{array}$ \\
\hline $\bar{c}_{1}\left(\mathrm{mmol} \mathrm{dm}^{-3}\right)$ & 0.6000 & 0.6000 & 0.6000 & 0.6000 & 0.6000 & 0.6000 & 0.6000 \\
\hline $\bar{c}_{2}\left(\mathrm{~mol} \mathrm{dm}^{-3}\right)$ & 0.5000 & 0.5000 & 0.5000 & 0.5000 & 0.5000 & 0.5000 & 0.5000 \\
\hline $\bar{c}_{3}\left(\mathrm{~mol} \mathrm{dm}^{-3}\right)$ & 0.5000 & 0.5001 & 0.5000 & 0.5000 & 0.5000 & 0.5000 & 0.5001 \\
\hline$\Delta c_{1}\left(\mathrm{mmol} \mathrm{dm}^{-3}\right)$ & 0.4000 & 0.0000 & 0.0000 & 0.2000 & 0.2000 & 0.0000 & 0.1334 \\
\hline$\Delta c_{2}\left(\mathrm{~mol} \mathrm{dm}^{-3}\right)$ & -0.00004 & 0.04818 & -0.00008 & 0.02425 & -0.00001 & 0.02405 & 0.01620 \\
\hline$\Delta c_{3}\left(\mathrm{~mol} \mathrm{dm}^{-3}\right)$ & 0.0000 & 0.0000 & 0.1169 & 0.0000 & 0.0584 & 0.0584 & 0.0399 \\
\hline $\mathrm{pH}_{\text {bot }}$ & 4.53 & 4.53 & 4.47 & 4.51 & 4.50 & 4.51 & 4.51 \\
\hline $\mathrm{pH}_{\text {top }}$ & 4.52 & 4.50 & 4.51 & 4.50 & 4.52 & 4.49 & 4.52 \\
\hline$d_{\text {bot }}\left(\mathrm{g} \mathrm{cm}^{-3}\right)$ & 1.034856 & 1.034712 & 1.036317 & 1.034786 & 1.035579 & 1.035514 & 1.035299 \\
\hline$d_{\text {top }}\left(\mathrm{g} \mathrm{cm}^{-3}\right)$ & 1.033204 & 1.033328 & 1.031714 & 1.033244 & 1.032459 & 1.032517 & 1.032751 \\
\hline$J_{\text {meas }}$ & 51.586 & 52.108 & 51.860 & 52.050 & 51.797 & 51.901 & 51.929 \\
\hline$J_{\text {calc }}$ & 51.587 & 52.071 & 51.836 & 52.031 & 51.751 & 51.931 & 52.024 \\
\hline$\Delta t(\mathrm{~s})$ & 40 & 22 & 14 & 34 & 36 & 14 & 28 \\
\hline$D_{\mathrm{A}, \text { meas }}\left(\times 10^{9} \mathrm{~m}^{2} \mathrm{~s}^{-1}\right)$ & 0.0963 & 0.6391 & 1.658 & 0.2011 & 0.2507 & 0.9720 & 0.3283 \\
\hline$D_{\mathrm{A}, \text { calc }}\left(\times 10^{9} \mathrm{~m}^{2} \mathrm{~s}^{-1}\right)$ & 0.0963 & 0.6392 & 1.657 & 0.2013 & 0.2509 & 0.9721 & 0.3283 \\
\hline
\end{tabular}

in ref 19. These include (a) baseline correction scans to correct for optical imperfections, done with bottom solution in both the diffusion and reference arms of the cell before sharpening the boundary; (b) fpf scans to determine the fractional part of the total fringe number accurately, done just after the sharpened boundary is allowed to diffuse; and (c) the 50 diffusion scans mentioned earlier. The magnification factor of the cylinder lens was 1.7602 .

\section{Results}

In Table 1, we report the results of the analysis of each individual experiment. In the table, $\bar{c}_{i}(i=1,2,3)$ represents the average concentration of each solute, and $\Delta c_{i}$ is the difference between the initial concentration of the bottom and top solutions for each solute. We also report $\mathrm{pH}_{\text {top }}$ and $\mathrm{pH}_{\text {bot }}$, and the densities $d_{\text {top }}$ and $d_{\text {bot }}$ of each prepared solution. Here, the subscripts "top" and "bot" respectively indicate the top and bottom solutions. In the same table, we list the measured total number of fringes $\left(J_{\text {meas }}\right)$ and its calculated value $\left(J_{\text {calc }}\right)$. The latter is obtained by fitting eq 6 to the $\left(J, \Delta c_{1}, \Delta c_{2}, \Delta c_{3}\right)$ data of the seven experiments. The time offset $\Delta t$ and both the calculated $^{17,23}$ and extrapolated ${ }^{23}$ values of the Rayleigh coefficient $D_{\mathrm{A}}$ are also included in the table.

Table 2 contains the results obtained by analyzing all seven experiments at the overall average concentrations $\overline{\bar{c}}_{i}$. For our small concentration differences, the densities are assumed to be linear in solute concentrations, in accordance with the following equation:

$$
d=\bar{d}+H_{1}\left(c_{1}-\overline{\bar{c}}_{1}\right)+H_{2}\left(c_{2}-\overline{\bar{c}}_{2}\right)+H_{3}\left(c_{3}-\overline{\bar{c}}_{3}\right)
$$

The values of the parameters $\bar{d}$ and $H_{i}=\left(\partial d / \partial c_{i}\right)_{c j, j \neq i}$ were obtained by least-squares calculations from the densities of all seven pairs of solutions.

The partial molar volumes $\bar{V}_{i}$ were calculated from eqs 8 and 9 of ref 27. Table 2 also contains the refractive index increments $R_{i}$ obtained from the previously noted eq 6 fit. The parameters $a_{i}, b_{i}$, and $s_{i}$ (and the corresponding eigenvalues, $\lambda_{i}$ ) were obtained by least-squares calculations, using eq 1 . Finally, the nine quaternary diffusion coefficients $D_{i j}^{\mathrm{Q}}$ are listed at the bottom of Table 2. The corresponding statistical error of each $D_{i j}$ coefficient is obtained from the standard errors of the $a_{i}, b_{i}$, $s_{i}$, and $R_{i}$ parameters by the propagation-of-error analysis. ${ }^{32}$

1. Error Analysis. We first examine the precision of the quaternary $D_{i j}^{\mathrm{Q}}$ coefficient obtained by Rayleigh interferometry. We then compare the precision of these current Rayleigh
TABLE 2: Overall System Data

\begin{tabular}{|c|c|}
\hline parameter & value \\
\hline \multicolumn{2}{|c|}{ overall average concentration } \\
\hline$\overline{\bar{c}}_{1}$ & $0.6000 \mathrm{mmol} \mathrm{dm}^{-3}$ \\
\hline$\overline{\bar{c}}_{2}$ & $0.5000 \mathrm{~mol} \mathrm{dm}^{-3}$ \\
\hline$\overline{\bar{c}}_{3}$ & $0.5000 \mathrm{~mol} \mathrm{dm}^{-3}$ \\
\hline average density, $\bar{d}$ & $1.034020 \mathrm{~g} \mathrm{~cm}^{-3}$ \\
\hline \multicolumn{2}{|l|}{$H$ parameter } \\
\hline$H_{1}$ & $4.121 \mathrm{~g} \mathrm{~mol}^{-1}$ \\
\hline $\mathrm{H}_{2}$ & $0.02904 \mathrm{~g} \mathrm{~mol}^{-1}$ \\
\hline$H_{3}$ & $0.03921 \mathrm{~g} \mathrm{~mol}^{-1}$ \\
\hline \multicolumn{2}{|l|}{ partial molar volume } \\
\hline $\bar{V}_{1}$ & $10210 \mathrm{~cm}^{3} \mathrm{~mol}^{-1}$ \\
\hline $\bar{V}_{2}$ & $165.6 \mathrm{~cm}^{3} \mathrm{~mol}^{-1}$ \\
\hline $\bar{V}_{3}$ & $19.28 \mathrm{~cm}^{3} \mathrm{~mol}^{-1}$ \\
\hline $\bar{V}_{0}$ & $18.062 \mathrm{~cm}^{3} \mathrm{~mol}^{-1}$ \\
\hline \multicolumn{2}{|c|}{ refractive index increment } \\
\hline$R_{1}$ & $129000 \mathrm{dm}^{3} \mathrm{~mol}^{-1}$ \\
\hline$R_{2}$ & $1080 \mathrm{dm}^{3} \mathrm{~mol}^{-1}$ \\
\hline$R_{3}$ & $444.3 \mathrm{dm}^{3} \mathrm{~mol}^{-1}$ \\
\hline \multicolumn{2}{|l|}{ eigenvalue } \\
\hline$\lambda_{1}$ & $0.0857 \times 10^{-9} \mathrm{~m}^{2} \mathrm{~s}^{-1}$ \\
\hline$\lambda_{2}$ & $0.547 \times 10^{-9} \mathrm{~m}^{2} \mathrm{~s}^{-1}$ \\
\hline$\lambda_{3}$ & $1.18 \times 10^{-9} \mathrm{~m}^{2} \mathrm{~s}^{-1}$ \\
\hline \multicolumn{2}{|l|}{ parameters from eq $1^{a}$} \\
\hline$a_{1}$ & $0.0357 \pm 0.0075$ \\
\hline$a_{2}$ & $0.133 \pm 0.016$ \\
\hline$a_{3}$ & $1.239 \pm 0.016$ \\
\hline$b_{1}$ & $0.051 \pm 0.012$ \\
\hline$b_{2}$ & $0.887 \pm 0.014$ \\
\hline$b_{3}$ & $-0.219 \pm 0.017$ \\
\hline$s_{1}$ & $291.1 \pm 1.1$ \\
\hline$s_{2}$ & $427.3 \pm 3.8$ \\
\hline$s_{3}$ & $1080.2 \pm 3.2$ \\
\hline \multicolumn{2}{|l|}{ quaternary coefficient } \\
\hline$D_{11}$ & $(0.08706 \pm 0.00053) \times 10^{9} \mathrm{~m}^{2} \mathrm{~s}^{-1}$ \\
\hline$D_{12}$ & $(0.000104 \pm 0.000012) \times 10^{9} \mathrm{~m}^{2} \mathrm{~s}^{-1}$ \\
\hline$D_{13}$ & $(0.0000911 \pm 0.0000078) \times 10^{9} \mathrm{~m}^{2} \mathrm{~s}^{-1}$ \\
\hline$D_{21}$ & $(4.00 \pm 0.56) \times 10^{9} \mathrm{~m}^{2} \mathrm{~s}^{-1}$ \\
\hline$D_{22}$ & $(0.5633 \pm 0.0077) \times 10^{9} \mathrm{~m}^{2} \mathrm{~s}^{-1}$ \\
\hline$D_{23}$ & $(0.0619 \pm 0.0040) \times 10^{9} \mathrm{~m}^{2} \mathrm{~s}^{-1}$ \\
\hline$D_{31}$ & $(8.1 \pm 2.1) \times 10^{9} \mathrm{~m}^{2} \mathrm{~s}^{-1}$ \\
\hline$D_{32}$ & $(0.160 \pm 0.022) \times 10^{9} \mathrm{~m}^{2} \mathrm{~s}^{-1}$ \\
\hline$D_{33}$ & $(1.163 \pm 0.010) \times 10^{9} \mathrm{~m}^{2} \mathrm{~s}^{-1}$ \\
\hline
\end{tabular}

measurements on the Gosting machine with the earlier Gouy measurements on the machine at the Naples facility.

2. Lysozyme Systems. We observe the following, in terms of the propagation-of-errors results:

(1) Errors of the main terms $D_{i i}^{\mathrm{Q}}$ are $\sim 0.5 \%-1.5 \%$ of $D_{i i}^{\mathrm{Q}}$ itself and are slightly larger than the corresponding ternary main terms $D_{i i}^{\mathrm{T}}$. 
TABLE 3: Comparisons of Quaternary Diffusion Coefficients of Lysozyme-Tetra(ethylene glycol) $-\mathrm{NaCl}-\mathrm{H}_{2} \mathrm{O}$, Calculated Using Different Sets of Experiments

\begin{tabular}{|c|c|c|c|c|c|}
\hline & all & all but $\mathrm{D}$ & all but $\mathrm{E}$ & all but $\mathrm{F}$ & all but $\mathrm{G}$ \\
\hline \multicolumn{6}{|c|}{ Quaternary Diffusion Coefficient, $D_{i j}\left(\times 10^{9} \mathrm{~m}^{2} \mathrm{~s}^{-1}\right)$} \\
\hline$D_{11}$ & $0.08706 \pm 0.00053$ & $0.08714 \pm 0.00072$ & $0.08692 \pm 0.00053$ & $0.08698 \pm 0.00056$ & $0.08701 \pm 0.00064$ \\
\hline$D_{12}$ & $0.000104 \pm 0.000012$ & $0.000104 \pm 0.000013$ & $0.000105 \pm 0.000011$ & $0.000106 \pm 0.000013$ & $0.000103 \pm 0.000014$ \\
\hline$D_{13}$ & $0.0000911 \pm 0.0000078$ & $0.0000897 \pm 0.0000094$ & $0.0000906 \pm 0.0000070$ & $0.0000901 \pm 0.0000089$ & $0.0000911 \pm 0.0000091$ \\
\hline$D_{21}$ & $4.00 \pm 0.56$ & $3.84 \pm 0.82$ & $4.16 \pm 0.57$ & $4.11 \pm 0.59$ & $4.06 \pm 0.69$ \\
\hline$D_{22}$ & $0.5633 \pm 0.0077$ & $0.5633 \pm 0.0086$ & $0.5636 \pm 0.0072$ & $0.5606 \pm 0.0091$ & $0.5638 \pm 0.0090$ \\
\hline$D_{23}$ & $0.0619 \pm 0.0040$ & $0.0623 \pm 0.0047$ & $0.0619 \pm 0.0036$ & $0.0617 \pm 0.0045$ & $0.0620 \pm 0.0046$ \\
\hline$D_{31}$ & $8.1 \pm 2.1$ & $8.8 \pm 3.1$ & $7.7 \pm 2.1$ & $7.6 \pm 2.2$ & $7.9 \pm 2.5$ \\
\hline$D_{32}$ & $0.160 \pm 0.022$ & $0.160 \pm 0.025$ & $0.159 \pm 0.021$ & $0.170 \pm 0.027$ & $0.159 \pm 0.026$ \\
\hline$D_{33}$ & $1.163 \pm 0.010$ & $1.163 \pm 0.012$ & $1.1634 \pm 0.0092$ & $1.165 \pm 0.011$ & $1.163 \pm 0.012$ \\
\hline \multicolumn{6}{|c|}{ Refractive Index Increment, $R_{i}\left(\mathrm{dm}^{3} \mathrm{~mol}^{-1}\right)$} \\
\hline $\begin{array}{l}R_{1} \\
R_{2} \\
R_{3}\end{array}$ & $\begin{array}{l}(1.2904 \pm 0.0012) \times 10^{5} \\
(1.0803 \pm 0.0010) \times 10^{3} \\
444.27 \pm 0.42\end{array}$ & $\begin{aligned}(1.2900 \pm 0.0013) & \times 10^{5} \\
(1.0805 & \pm 0.0011) \times 10^{3} \\
444.12 \pm 0.47 & \end{aligned}$ & $\begin{aligned}(1.2903 \pm 0.0015) & \times 10^{5} \\
(1.0802 \pm 0.0012) & \times 10^{3} \\
444.27 \pm 0.42 & \end{aligned}$ & $\begin{aligned} &(1.2903 \pm 0.0014) \times 10^{5} \\
&(1.0806 \pm 0.0012) \times 10^{3} \\
& 444.30 \pm 0.49\end{aligned}$ & $\begin{array}{l}(1.29084 \pm 0.00075) \times 10^{5} \\
(1.08066 \pm 0.00062) \times 10^{3} \\
444.41 \pm 0.26\end{array}$ \\
\hline
\end{tabular}

(2) The largest errors are in those cross-term diffusion coefficients $D_{i j}^{\mathrm{Q}}(i \neq j)$ that correspond to the motion of small solutes, because of the concentration gradient of the largest ones. This same behavior is found in the ternary systems. ${ }^{3,33}$

(3) The errors in these quaternary cross-term coefficients are always larger than the analogous ones from the corresponding ternaries. ${ }^{3,33}$ They can also reach $\sim 25 \%$ of $D_{i j}^{\mathrm{Q}}$. We also observe large errors in the cross-term coefficients for the motion of the largest molecule (lysozyme), because of the concentration gradients of the small ones. This probably depends on numerical problems that are encountered in obtaining the very small values of these coefficients.

3. Comparison with the Earlier Four-Component System. For a proper comparison of our Rayleigh results with the earlier Gouy interferometric four-component system of cyclodextrinphenylalanine-monobutylurea- $\mathrm{H}_{2} \mathrm{O}$, we must use the same method of estimating the errors.

When the Gouy system was analyzed in $1992,{ }^{17}$ the propagation-of-error equations, which include 93 nonzero derivatives, had not yet been derived. Consequently, the precision of each $D_{i j}$ coefficient was estimated as follows. Data for one or more experiments, in turn, were deleted, always keeping the three $\alpha_{i}$ $=1$ cases. ${ }^{17,20}$ The $D_{i j}$ coefficients were then calculated for each set by the previously described nonlinear least-squares procedure. There were six such sets, including the set of all eight experiments. The estimated error for each $D_{i j}$ coefficient was taken as the standard error of the mean of its values from the six sets.

A similar analysis of our current Rayleigh results was obtained by removing, one at a time, the following data sets from the full set of seven, keeping the three $\alpha_{i}=1$ cases, as was done with the Gouy data: $\mathrm{D}(1 / 2,1 / 2,0), \mathrm{E}(1 / 2,0,1 / 2), \mathrm{F}\left(0,{ }_{1} / 2,1 / 2\right)$, or $\mathrm{G}(1 / 3,1 / 3,1 / 3)$, where the parentheses contain the $\alpha_{i}$ values. The results from these four cases, plus the full set of seven, are given in Table 3.

Table 3 shows that, for each $D_{i j}^{\mathrm{Q}}$ coefficient, the differences between its value for the complete set of seven experiments and its values from each of the four six-experiment sets are always within one standard deviation of the complete set. Moreover, these differences are generally very small, except for $D_{21}^{\mathrm{Q}}$ and $D_{31}^{\mathrm{Q}}$ and, in one case, for $D_{32}^{\mathrm{Q}}$ (the "all but F" case). In these cases, the differences are as follows: $1.5 \%-4 \%$ for $D_{21}^{\mathrm{Q}}$, with a corresponding relative standard error of $14 \%$; $2.5 \%-8 \%$ for $D_{31}^{\mathrm{Q}}$, with a corresponding relative standard error of $26 \%$; and $6 \%$ for $D_{32}^{\mathrm{Q}}$, with a corresponding relative standard error of $14 \%$. These relative standard errors are found in Table 4; they are denoted by $\%$ SE, are equal to $100 \times$ (the standard error from propagation of error equations $) / D_{i j}^{\mathrm{Q}}$, and are based on the complete set.

These results confirm the general rule that the largest variations are observed for the cross-term diffusion coefficients $D_{i j}^{\mathrm{Q}}$ for which the ratio $R_{j} / R_{i}$ is larger than unity. The $R_{i}$ parameter and the ratios $R_{j} / R_{i}$ are in Table 4 , as are the eigenvalues $\lambda_{i}$. We also observe that the $D_{i j}^{\mathrm{Q}}$ errors do not increase much in going from seven to six experiments.

Some idea of the improved precision from the Gosting diffusiometer for four-component systems may now be obtained from a comparison with the earlier Gouy results. ${ }^{17}$ Table 4 contains, for each of the nine $D_{i j}^{\mathrm{Q}}$ coefficients, the standard error of the mean of its values from all six sets of Gouy experiments for cyclodextrin-phenyalanine-monobutylurea$\mathrm{H}_{2} \mathrm{O}$ and the percentage relative standard deviation of the corresponding mean for this system. ${ }^{17}$ Our corresponding Rayleigh results from the Gosting machine for lysozyme-tetra(ethylene glycol) $-\mathrm{NaCl}-\mathrm{H}_{2} \mathrm{O}$ are also included, as well as the propagation-of-error and relative propagation-of-error results. We note that that the standard-error-of-the-mean results are significantly smaller than the propagation-of-error results. This implies a very consistent series of experiments.

The error comparison for the two systems is based on a common treatment of the data, namely, averaging the $D_{i j}^{\mathrm{Q}}$ coefficients from various combinations of experiment sets and calculating the standard deviation of the mean. It cannot really be completely correct because the two systems are different, and relative values of the $D_{i j}, \lambda_{i}$, and $R_{i}$ can significantly influence the least-squares fitting errors. In particular, the mainterm errors depend on $D_{i j}$ and $\lambda_{i}$, and the cross-term errors depend on $D_{i j}, \lambda_{i}$, and $R_{i}$. When the ratio $R_{j} / R_{i}$ is large, errors in the $D_{i j}$ are also large. ${ }^{17}$ Furthermore, convergence is better and errors are smaller when the eigenvalues $\lambda_{i}$ are significantly different. This is the case here but is not so for the earlier Gouy system, where $\lambda_{2}$ and $\lambda_{3}$ are very close (cf. Table 4). Finally, the differing difficulties of solution preparation also contribute to differences in errors. Nonetheless, the percentage relative errors from the Gosting-machine Rayleigh results are significantly smaller than those for the Gouy results. This is particularly true for the cross terms, even though the ratios $R_{1} / R_{2}$ and $R_{1} / R_{3}$ are considerably larger than those for the earlier Gouy system. This suggests that the automated Gosting diffusiometer and its Rayleigh procedures have significantly improved the relative precision of determining the cross terms and probably all terms. 
TABLE 4: Comparison of Four-Component $D_{i j}\left(10^{9} \mathrm{~m}^{2} \mathrm{~s}^{-1}\right)$ Errors on the Same Basis ${ }^{a}$

\begin{tabular}{|c|c|c|c|c|c|c|c|c|}
\hline & \multicolumn{3}{|c|}{$\mathrm{CD}-\mathrm{PA}-\mathrm{MBU}-\mathrm{H}_{2} \mathrm{O}^{b}$} & \multicolumn{3}{|c|}{ lysozyme-PEG4- $\mathrm{NaCl}-\mathrm{H}_{2} \mathrm{O}$} & \multirow[b]{2}{*}{ SE from POE } & \multirow[b]{2}{*}{$\% \mathrm{SE}$} \\
\hline & $D_{i j}$ & SD mean & $\%$ SD mean & $D_{i j}$ & SD mean & $\% \mathrm{SD}$ mean & & \\
\hline$D_{11}$ & 0.316 & 0.001 & 0.32 & 0.0871 & 0.00009 & 0.10 & 0.00053 & 0.61 \\
\hline$D_{12}$ & -0.001 & 0.001 & 100.0 & 0.00010 & 9. $\times 10^{-7}$ & 0.91 & $1.16 \times 10^{-5}$ & 110.2 \\
\hline$D_{13}$ & -0.001 & 0.0001 & 10.0 & 0.000091 & $6 . \times 10^{-7}$ & 0.678 & $0.78 \times 10^{-5}$ & 8.6 \\
\hline$D_{21}$ & -0.132 & 0.030 & 22.7 & 4.004 & 0.124 & 3.10 & 0.561 & 14.0 \\
\hline$D_{22}$ & 0.646 & 0.013 & 2.0 & 0.563 & 0.0013 & 0.232 & 0.0077 & 1.37 \\
\hline$D_{23}$ & 0.064 & 0.003 & 4.7 & 0.0619 & 0.00021 & 0.339 & 0.00403 & 6.51 \\
\hline$D_{31}$ & -0.076 & 0.061 & 80.3 & 8.104 & 0.465 & 5.74 & 2.05 & 25.1 \\
\hline$D_{32}$ & 0.001 & 0.021 & 2100 & 0.1603 & 0.0047 & 2.94 & 0.0225 & 14.02 \\
\hline$D_{33}$ & 0.632 & 0.003 & 0.47 & 1.163 & 0.00074 & 0.064 & 0.0102 & 0.88 \\
\hline$\lambda_{1}$ & 0.3151 & & & 0.0851 & & & & \\
\hline$\lambda_{2}$ & 0.6294 & & & 0.5477 & & & & \\
\hline$\lambda_{3}$ & 0.6492 & & & 1.1801 & & & & \\
\hline$R_{1}$ & 139.5 & & & 129045 & & & & \\
\hline$R_{2}$ & 36.51 & & & 1080 & & & & \\
\hline$R_{3}$ & 17.30 & & & 444.3 & & & & \\
\hline$R_{1} / R_{2}$ & 3.8 & & & 119.5 & & & & \\
\hline$R_{1} / R_{3}$ & 8.1 & & & 290.4 & & & & \\
\hline$R_{2} / R_{3}$ & 2.1 & & & 2.4 & & & & \\
\hline
\end{tabular}

${ }^{a} D_{i j}$ are the coefficient values from all the experiments. SD mean is the standard error of the mean; \% SD mean is $100 \times(\mathrm{SD}$ mean $) / D_{i j}$. SE from $\mathrm{POE}$ is the standard error of the coefficient from the propagation-of-error calculation; $\% \mathrm{SE}$ is $100 \times\left(\mathrm{SE}\right.$ from POE)/Dij. Units of all $D_{i j}$ and $\lambda_{i}$ are $10^{9} \mathrm{~m}^{2} \mathrm{~s}^{-1} \cdot{ }^{b} \alpha$-cyclodextrin-L-phenylalanine-monobutylurea- $\mathrm{H}_{2} \mathrm{O} .{ }^{14}$

\section{Discussion of Diffusion Coefficients}

The superscripts Q (quaternary), T (ternary), and B (binary), where $D_{i i}^{\mathrm{B}}=D_{i}$, have been used to distinguish the various coefficients in the following discussion.

It is possible to envision the properties of the diffusion coefficients in terms of viscosity, electrostatic, obstruction, and excluded volume effects. The mixing of these macroscopic and microscopic concepts is not exact. For example, a direct connection between viscosity and diffusion is forbidden by the "Curie principle" of irreversible thermodynamics, because they are of different tensor orders. ${ }^{34-36}$ However, these concepts often provide a useful picture and qualitative approximations of the four-component quantities, on the basis of binary or ternary data, as will be seen below.

1. Quaternary Diffusion Coefficients. In Table 2, we observe the following, for the cross-term diffusion coefficients:

(1) All the cross-term diffusion coefficients are positive.

(2) The $D_{12}^{\mathrm{Q}}$ and $D_{13}^{\mathrm{Q}}$ values are very small (between $10^{-13}$ and $10^{-14} \mathrm{~m}^{2} \mathrm{~s}^{-1}$ ).

(3) The $D_{23}^{\mathrm{Q}}$ and $D_{32}^{\mathrm{Q}}$ values are small (between $10^{-10}$ and $\left.10^{-11} \mathrm{~m}^{2} \mathrm{~s}^{-1}\right)$.

(4) The $D_{21}^{\mathrm{Q}}$ and $D_{31}^{\mathrm{Q}}$ values are quite large $\left(\sim 5 \times 10^{-9}\right.$ $\mathrm{m}^{2} \mathrm{~s}^{-1}$ ), and both are larger than all the main-term diffusion coefficients.

The corresponding ternary system without PEG4 exhibits similar behavior: ${ }^{3} D_{13}^{\mathrm{T}}$ is very small, $D_{31}^{\mathrm{T}}$ is very large, and $D_{31}^{\mathrm{T}}$ is larger than both main-term diffusion coefficients $D_{11}^{\mathrm{T}}$ and $D_{33}^{\mathrm{T}}$.

On the basis of published and unpublished data for several similar three- and four-component systems, cross-term diffusion coefficients for the motion of a large molecule due to the concentration gradient of a much smaller one are almost negligible. On the other hand, cross-term diffusion coefficients for the motion of a small molecule due to the concentration gradient of a much bigger one can be quite large. In intermediate cases, cross-term diffusion coefficients increase as the difference in size of the two solutes increases. ${ }^{37-40}$ This behavior is essentially due to an excluded volume effect of the large molecule on the small one, ${ }^{37-38}$ combined with the choice of the molar concentration scale that does not take into account the big difference in the solute molecular weight.

2. Effect of PEG4 on Lysozyme-NaCl Coupled Transport. We now consider the effects of PEG4 on the diffusion coefficients $D_{11}^{\mathrm{Q}}, D_{33}^{\mathrm{Q}}, D_{13}^{\mathrm{Q}}$, and $D_{31}^{\mathrm{Q}}$.

2.1. $D_{11}^{Q}$. The value of the $D_{11}^{Q}$ coefficient is $(0.0870 \pm$ $0.0005) \times 10^{-9} \mathrm{~m}^{2} \mathrm{~s}^{-1}$. The ternary diffusion coefficient for the system lysozyme $(1)-\mathrm{NaCl}(3)-\mathrm{H}_{2} \mathrm{O}$ is $D_{11}^{\mathrm{T}}=(0.1182 \pm$ $0.0005) \times 10^{-9} \mathrm{~m}^{2} \mathrm{~s}^{-1}$, whereas the corresponding binary diffusion coefficient for lysozyme(1)-water is $D_{11}^{\mathrm{B}}=0.5508$ $\times 10^{-9} \mathrm{~m}^{2} \mathrm{~s}^{-1} 3$

The dramatic decrease from the value of $D_{11}^{\mathrm{B}}$ to the value of $D_{11}^{\mathrm{T}}$ is a consequence of the electrostatic coupling of the motions between ionic species. The diffusion coefficient of electrolytes in aqueous binary systems depends on the diffusivities (mobilities) of both ions and their electrostatic interaction. Because the ions are not independent of each other, the faster ion speeds up the slower one; conversely, the slower ion slows down the faster one. The experimental diffusion coefficients of the salt is then a combination of the interconnected diffusivities of both ions. ${ }^{3}$

This same situation also occurs in binary aqueous solutions of polyelectrolytes or charged proteins. The only difference is that, in this case, the number $z_{p}$ of low-molecular-weight dragging ions (the counterions) is large. Furthermore, the difference between the diffusivity of the charged protein and its counterions is also large, with the protein diffusivity being much smaller. However, adding $\mathrm{NaCl}$ to the protein solution decreases the electrostatic potential between the polyions and the counterions, so the dragging effect also decreases. Consequently, $D_{11}^{\mathrm{T}}$ is more similar to the value of the protein diffusivity by itself, and, thus, is much smaller than the $D_{11}^{\mathrm{B}}$ value.

The decrease from the values of $D_{11}^{\mathrm{T}}$ to the value of $D_{11}^{\mathrm{Q}}$ can be simply interpreted as a consequence of an increase of the solution viscosity from the added PEG4. ${ }^{33,39}$ This is predicted by the Stokes-Einstein equation applied to microscopic systems. Indeed, the ratio between the lysozyme main diffusion coefficient in the presence and absence of PEG is $D_{11}^{\mathrm{Q}} / D_{11}^{\mathrm{T}}=0.736$ \pm 0.007 , whereas the inverse ratio of the corresponding 
TABLE 5: Diffusion and Viscosity Ratios

\begin{tabular}{ccc}
\hline diffusion ratio & viscosity ratio \\
\hline \multicolumn{4}{c}{ Component 1} \\
$D_{11}^{\mathrm{Q}} / D_{11}^{\mathrm{T}}=0.736 \pm 0.007$ & $1 /\left(\eta^{\mathrm{Q}} / \eta^{\mathrm{T}}\right)=0.752 \pm 0.008$ \\
$D_{11}^{\mathrm{T}} / D_{11}^{\mathrm{B}}=0.215 \pm 0.001$ & $1 /\left(\eta^{\mathrm{T}} / \eta_{1}^{\mathrm{B}}\right)=0.972 \pm 0.003$ \\
$D_{11}^{\mathrm{Q}} / D_{11}^{\mathrm{B}}=0.158 \pm 0.001$ & $1 /\left(\eta^{\mathrm{Q}} / \eta_{1}^{\mathrm{B}}\right)=0.682 \pm 0.005$ \\
\multicolumn{5}{c}{ Component 3 } \\
$D_{33}^{\mathrm{Q}} / D_{33}^{\mathrm{T}}=0.797 \pm 0.007$ & $1 /\left(\eta^{\mathrm{Q}} / \eta^{\mathrm{T}}\right)=0.752 \pm 0.008$ \\
$D_{33}^{\mathrm{T}} / D_{33}^{\mathrm{B}}=0.980 \pm 0.001$ & $1 /\left(\eta^{\mathrm{T}} / \eta_{3}^{\mathrm{B}}\right)=0.977 \pm 0.002$ \\
$D_{33}^{\mathrm{Q}} / D_{33}^{\mathrm{B}}=0.787 \pm 0.001$ & $1 /\left(\eta^{\mathrm{Q}} / \eta_{3}^{\mathrm{B}}\right)=0.734 \pm 0.010$ \\
$D_{22}^{\mathrm{Q}} / D_{22}^{\mathrm{B}}=0.932 \pm 0.014$ & $1 /\left(\eta^{\mathrm{Q}} / \eta_{2}^{\mathrm{B}}\right)=0.952 \pm 0.006$
\end{tabular}

viscosities is similar, with $\eta^{\mathrm{T}} / \eta^{\mathrm{Q}}=0.752 \pm 0.008$. (See Table 5.) Here, the Stokes-Einstein equation is quite successful in solutions with a relatively low concentration of macromolecules. This strong correlation between diffusion and viscosity is generally observable when the diffusing species (i.e., the protein) is large, with respect to those other components of the system (i.e., $\mathrm{H}_{2} \mathrm{O}, \mathrm{NaCl}$, and PEG4) that can be approximated as a continuum. However, when electrostatic coupling occurs, viscosity poorly correlates with diffusion, and the difference between $D_{11}^{\mathrm{B}}$ and $D_{11}^{\mathrm{T}}$ cannot be predicted from the viscosity behavior.

2.2. $D_{33}^{Q}$. The value of the $D_{33}^{\mathrm{Q}}$ coefficient is $(1.16 \pm 0.01) \times$ $10^{-9} \mathrm{~m}^{2} \mathrm{~s}^{-1}$. The ternary diffusion coefficient for the system lysozyme $(1)-\mathrm{NaCl}(3)-\mathrm{H}_{2} \mathrm{O}$ is $D_{33}^{\mathrm{T}}=(1.445 \pm 0.001) \times 10^{-9}$ $\mathrm{m}^{2} \mathrm{~s}^{-1}$, whereas the corresponding binary diffusion coefficient for $\mathrm{NaCl}(3)-\mathrm{H}_{2} \mathrm{O}$ is $D_{33}^{\mathrm{B}}=1.4740 \times 10^{-9} \mathrm{~m}^{2} \mathrm{~s}^{-1} .{ }^{41}$

The small decrease of the $\mathrm{NaCl}$ main-term diffusion coefficient observed by adding lysozyme to $\mathrm{NaCl}(3)-\mathrm{H}_{2} \mathrm{O}$ can be explained by a lysozyme obstruction effect. This effect is very small, because the lysozyme concentration is small. The further decrease of $D_{33}^{\mathrm{T}}$ to $D_{33}^{\mathrm{Q}}$ on adding PEG4 to the ternary lysozyme(1) $-\mathrm{NaCl}(3)-\mathrm{H}_{2} \mathrm{O}$ system may be related to a $\mathrm{PEG}$ obstruction effect on the motion of the simple salt. This further decrease is larger because the volume fraction occupied by PEG4 in solution is larger than that of lysozyme (the volume fraction $\Phi_{i}\left(\Phi_{i}=c_{i} V_{i}\right.$, with $V_{i}$ given in $\left.\mathrm{dm}^{3} \mathrm{~mol}^{-1}\right)$ for PEG4 is $\Phi_{\mathrm{PEG} 4} \cong 0.1$ and for lysozyme is $\Phi_{\text {lys }} \cong 0.01$ ). Here, the viscosity quantitatively fails to predict the change of this diffusion coefficient. This is not a surprise, considering the small size of both the $\mathrm{Na}^{+}$and $\mathrm{Cl}^{-}$ions (i.e., the continuum approximation fails).

2.3. $D_{13}^{Q}$. The quaternary $D_{13}^{\mathrm{Q}}$ coefficient, which is equal to $(0.0000911 \pm 0.0000078) \times 10^{-9} \mathrm{~m}^{2} \mathrm{~s}^{-1}$, is smaller than the corresponding ternary $D_{13}^{\mathrm{T}}\left(D_{13}^{\mathrm{T}}=(0.0001070 \pm 0.0000002) \times\right.$ $\left.10^{-9} \mathrm{~m}^{2} \mathrm{~s}^{-1}\right)$. This cross-term diffusion coefficient is proportional to the protein mobility; therefore, we compare the ratio between it in the presence and absence of PEG4, $D_{13}^{\mathrm{Q}} / D_{13}^{\mathrm{T}}=$ $0.85 \pm 0.09$, with the inverse ratio of the corresponding viscosities, $\eta^{\mathrm{T}} / \eta^{\mathrm{Q}}=0.752 \pm 0.008$. Again, the behavior of diffusion and viscosity are in reasonable agreement and within the experimental error.

2.4. $D_{31}^{Q}$. The quaternary $D_{31}^{\mathrm{Q}}$ coefficient, which is equal to $(8.1 \pm 2.1) \times 10^{-9} \mathrm{~m}^{2} \mathrm{~s}^{-1}$, is smaller than the corresponding ternary $D_{31}^{\mathrm{T}}\left(D_{31}^{\mathrm{T}}=(13.8 \pm 0.2) \times 10^{-9} \mathrm{~m}^{2} \mathrm{~s}^{-1}\right){ }^{3}$ Therefore, adding PEG4 seems to decrease the effect of a lysozyme concentration gradient on the motion of the simple salt. This does not seem to strictly depend on the variation of the $\mathrm{NaCl}$ main-term diffusion coefficient. In fact, we have $D_{31}^{\mathrm{Q}} / D_{33}^{\mathrm{Q}}=$ $7.0 \pm 1.9$, whereas $D_{31}^{\mathrm{T}} / D_{33}^{\mathrm{T}}=9.6 \pm 0.2$.
The number of $\mathrm{NaCl}$ molecules moved by a single lysozyme molecule also reflects this effect. This value can be obtained as follows. If we write the flux equation for both lysozyme and $\mathrm{NaCl}$, we have

$$
\left\{\begin{array}{l}
J_{1}^{\mathrm{Q}}=-D_{11}^{\mathrm{Q}} \nabla c_{1}-D_{12}^{\mathrm{Q}} \nabla c_{2}-D_{13}^{\mathrm{Q}} \nabla c_{3} \\
J_{3}^{\mathrm{Q}}=-D_{31}^{\mathrm{Q}} \nabla c_{1}-D_{32}^{\mathrm{Q}} \nabla c_{2}-D_{33}^{\mathrm{Q}} \nabla c_{3}
\end{array}\right.
$$

In the absence of PEG4 and $\mathrm{NaCl}$ concentration gradients, eq 9 reduces to

$$
\left\{\begin{array}{l}
J_{1}^{\mathrm{Q}}=-D_{11}^{\mathrm{Q}} \nabla c_{1} \\
J_{3}^{\mathrm{Q}}=-D_{31}^{\mathrm{Q}} \nabla c_{1}
\end{array}\right.
$$

and then $J_{3}^{\mathrm{Q}} / J_{1}^{\mathrm{Q}}=D_{31}^{\mathrm{Q}} / D_{11}^{\mathrm{Q}}$. This shows that the flux of $\mathrm{NaCl}$ per unit flux of lysozyme equals the ratio of the cross-term diffusion coefficient of $\mathrm{NaCl}$, because of the lysozyme concentration gradient and the lysozyme main-term diffusion coefficient. The values of this ratio obtained for the quaternary and ternary systems are, respectively, $D_{31}^{\mathrm{Q}} / D_{11}^{\mathrm{Q}}=93 \pm 25$ and $D_{31}^{\mathrm{T}} / D_{11}^{\mathrm{T}}=117.2 \pm 2$, with a modest decrease for the quaternary of the number of $\mathrm{NaCl}$ molecules moved by lysozyme. This diffusion coefficient is the largest one, both in ternary ${ }^{3,33}$ and quaternary systems, and indicates the large effect of the protein molecule on the motion of the simple salt. The physical reason for such a large effect is the exclusion of the salt ions from the volume occupied by the protein, because of hard core repulsive force, as first proposed in ref 3 and later formalized in predictive equations. ${ }^{37,42}$

3. Coupled Transport Directly Related to PEG4. We now analyze the quaternary diffusion coefficient related directly to the motion of PEG4.

3.1. $D_{22}^{Q}$. The value of the $D_{22}^{\mathrm{Q}}$ coefficient is $(0.563 \pm 0.008)$ $\times 10^{-9} \mathrm{~m}^{2} \mathrm{~s}^{-1}$, whereas the corresponding binary diffusion coefficient of PEG4(2) $-\mathrm{H}_{2} \mathrm{O}$ is $D_{22}^{\mathrm{B}}=0.6042 \times 10^{-9} \mathrm{~m}^{2} \mathrm{~s}^{-1} .{ }^{43}$ The protein volume fraction is relatively low, and electrostatic coupling does not occur; therefore, we expect that the effect of protein on the main diffusion coefficient $D_{22}^{\mathrm{Q}}$ is not significant. We also expect that the effect of the smaller $\mathrm{NaCl}$ on PEG4 transport is essentially related to viscosity. ${ }^{33}$ The prediction of $D_{22}^{\mathrm{Q}}$ from $D_{22}^{\mathrm{B}}$, using the corresponding viscosities, ${ }^{39}$ is fairly good.

3.2. Other Cross-Term Diffusion Coefficients. The quaternary $D_{21}^{\mathrm{Q}}$ coefficient, although smaller than the $D_{31}^{\mathrm{Q}}$ coefficient, is large and of the same order of magnitude. The ratio $D_{21}^{\mathrm{Q}} / D_{11}^{\mathrm{Q}}=46.0 \pm 7.2$, which indicates that substantial amounts of PEG4 are moved by a single lysozyme molecule, just as $\mathrm{NaCl}$ was.

However, the most interesting thing is the analysis of the ratios $D_{i l}^{\mathrm{Q}} / D_{i i}^{\mathrm{Q}}$. In fact, these ratios are very similar for PEG4 and $\mathrm{NaCl}: D_{21}^{\mathrm{Q}} / D_{22}^{\mathrm{Q}}=7.1 \pm 1.2$ and $D_{31}^{\mathrm{Q}} / D_{33}^{\mathrm{Q}}=7.0 \pm 1.9$. This implies that, besides the different nature of the two solutes, the effect of lysozyme on both their motions is exactly the same. This is possible only for a nonspecific effect, such as the excluded volume effect.

4. Comments on Cross-Term Effects. When considering the magnitudes of the diffusion coefficients, we are also interested in differences in the flows with and without the cross terms. As noted in the Introduction, cross terms can have a significant effect on the nucleation and crystal growth of proteins, because these processes are sensitive to small concentration differences. 
Depending on the gradients, the large values of $D_{21}$ and $D_{31}$ typically result in flow differences of $\sim 10 \%-15 \%$, compared to setting them equal to zero. Clearly, the nonzero cross terms do not permit a constant gradient of nonprotein solutes to remain, even if they start out that way. ${ }^{44,45}$ The time-dependent transport of noticeable amounts of PEG4 and $\mathrm{NaCl}$ by a lysozyme gradient can involve inclusion of nonprotein solutes and water in the protein crystal, alter the nucleation and precipitation processes and rates, create stress in the crystal, and degrade crystal quality.

\section{Conclusions}

Rayleigh interferometry has been successfully applied for the first time to determine precision mutual diffusion coefficients in quaternary systems, using an instrument that was designed and assembled by Gosting and co-workers ${ }^{18}$ and automated by M. Zeidler and some of the current authors (JGA and DGM). ${ }^{27}$ The precision of the experimental apparatus and the data processing is tested using the quaternary system lysozymetetra(ethylene glycol) $-\mathrm{NaCl}-\mathrm{H}_{2} \mathrm{O}$. Several assessments of the quality and statistics were used to examine the data processing and precision of the apparatus. The precision seems significantly better than that from the earlier interferometric study. ${ }^{17}$

The system investigated is of interest in the field of protein crystal growth, because lysozyme is a model protein in crystallogenesis and poly(ethylene glycol) (PEG) and $\mathrm{NaCl}$ are widely used as crystallizing agents. The comparison of these high-precision diffusion results for this quaternary system, along with its corresponding ternary ${ }^{3,33}$ and binary systems, ${ }^{3,28,43}$ has provided information on the effect of adding PEG to proteinsalt systems. The excluded volume effect and viscosity seem to be the most significant contributions to the action of PEG, although dielectric effects cannot be neglected.

The significant effects of large cross terms indicate that crystallization modeling using a pseudo-binary protein diffusion coefficient can lead to seriously misleading results.

The investigated system contains a monodisperse PEG oligomer, whereas polydisperse PEG oligomers are typically used in crystallization kits. Therefore, the present analysis will be extended to analogous protein systems containing PEG at higher molecular weights, which are polydisperse but will still have a narrow distribution of diffusion coefficients, as a function of the molecular weight.

Acknowledgment. This research was supported by an ASI (Italian Space Agency) contract and the NASA BioTechnology Program (through Grant No. NAG8-1356).

\section{References and Notes}

(1) Vergara, A.; Lorber, B.; Zagari, A.; Giegé, R. Acta Crystallogr., Sect. D: Biol. Crystallogr. 2003, 59, 2-15. (Topical review.)

(2) Robert, M.-C.; Vidal, O.; Garcia-Ruiz, J. M.; Otalora, F. Crystallization in Gels and Related Methods. In Crystallization of Nucleic Acids and Proteins: A Practical Approach; Ducruix, A., Giegé, R., Eds.; IRL Press: Oxford, U.K., 1999; pp 149-176.

(3) Albright, J. G.; Annunziata, O.; Miller, D. G.; Paduano, L.; Pearlstein, J. J. Am. Chem. Soc. 1999, 121, 3256-3266.

(4) Annunziata, O.; Paduano, L.; Pealrstein, A. J.; Miller, D. G.; Albright, J. G. J. Am. Chem. Soc. 2000, 122, 5916-5928
(5) Paduano, L.; Annunziata, O.; Pearlstein, A. J.; Miller, D. G.; Albright, J. G. J. Cryst. Growth 2001, 232, 149-156. 1389.

(6) Vergara, A.; Paduano, L.; Sartorio, R. Macromolecules 2002, 35 ,

(7) Capuano, F.; Vergara, A.; Paduano, L.; Annunziata, O.; Sartorio, R. "Protein Diffusion in Solutions Crowded by Poly(ethylene glycol)", manuscript in preparation.

(8) Vergara, A.; Paduano, L.; Vitagliano, V.; Sartorio, R. J. Phys. Chem. B 1999, 102, 8756 .

(9) Vergara, A; Paduano, L.; Mangiapia, G.; Sartorio, R. J. Phys. Chem. B 2001, 105, 11044.

(10) Mangiapia, G.; Paduano, L.; Vergara, A.; Sartorio, R. "A Novel Approach for Determining Diffusion Coefficients in Ternary System Containing a Polydisperse Solute. Applications to the Gouy Interferometry", submitted to J. Phys. Chem.

(11) Rai, G. P.; Cullinan, H. T., Jr. J. Chem. Eng. Data 1973, 18 , 213.

(12) Noulty, R. A.; Leaist, D. G. J. Phys. Chem. 1987, 91, 1655.

(13) Leaist, D. G. J. Chem. Soc., Faraday Trans. 1 1987, 83, 829.

(14) Leaist, D. G.; Hao, L. J. Solution Chem. 1993, 22, 263.

(15) Leaist, D. G. Ber. Bunsen-Ges. 1991, 95, 119.

(16) Leaist, D. G. J. Solution Chem. 1987, 16, 805-812.

(17) Paduano, L.; Sartorio, R.; Vitagliano, V.; Albright, J. G.; Miller,

D. G. J. Phys. Chem. 1992, 96, 7478.

(18) Gosting, L. J.; Kim, H.; Loewenstein, M. A.; Reinfeldsa, G.; Revzin, A. Rev. Sci. Instrum. 1973, 44, 1602.

(19) Miller, D. G.; Albright, J. G.; Mathew, R.; Lee, C. M.; Rard, J. A.; Eppstein, L. B. J. Phys. Chem. 1993, 97, 3885.

(20) Miller, D. G.; Paduano, L.; Sartorio, R.; Albright, J. G. J. Phys. Chem. 1994, 98, 13745.

(21) Miller, D. G.; Sartorio, R.; Paduano, L.; Rard, J. A.; Albright, J. G. J. Solution Chem. 1996, 25, 1185.

(22) Kim, H. J. Phys. Chem. 1966, 70, 562.

(23) Kim, H. J. Phys. Chem. 1969, 73, 1716

(24) Miller, D. G. J. Phys. Chem. 1988, 92, 4222.

(25) Miller, D. G. In International Symposium on Materials Handling in Pyrometallurgy; Purdy, G. R., Ed.; Proceedings of the Metallurgical Society of the Canadian Institute of Mining and Metallurgy, Vol. 18; Pergamon Press: New York, 1990; pp 29-40.

(26) Creeth, J. M. J. Am. Chem. Soc. 1955, 77, 6428.

(27) Rard, J. A.; Miller, D. G.; Albright, J. G.; Zeidler, M. E. J. Chem. Soc., Faraday Trans. 1996, 93, 4187.

(28) Rard, J. A. J. Chem. Thermodyn. 1996, 28, 83.

(29) Weast, R. C., Ed. CRC Handbook of Chemistry and Physics, 57th ed.; CRC Press: Boca Raton, FL, 1975; p B-165.

(30) See ref 29, p F-51.

(31) Philpot, J. St. L.; Cook, G. H. Research (London) 1948, 1, 234.

(32) Wentworth, W. E. J. Chem. Educ. 1965, 42, 96.

(33) Vergara, A.; Paduano, L.; Sartorio, R.; Annunziata, O.; Miller, D. G.; Albright, J. G. "Multicomponent Diffusion in Crowded Solutions. 2. Mutual Diffusion in the Ternary System Tetra(ethylene glycol) $-\mathrm{NaCl}-$ Water", submitted to J. Phys. Chem. B.

(34) DeGroot, S. R.; Mazur, P. Non-Equilibrium Thermodynamics; Interscience: New York, 1962.

(35) Miller, D. G. J. Phys. Chem. 1960, 64, 1598

(36) Miller, D. G. J. Phys. Chem. 1966, 70, 2639.

(37) Vergara, A.; Paduano, L.; Vitagliano, V.; Sartorio, R. J. Phys. Chem. B 2000, 104, 8086-8074.

(38) Vergara, A.; Paduano, L.; Vitagliano, V.; Sartorio, R. Mater. Chem. Phys. 2000, 66, 126-131.

(39) Vergara, A.; Paduano, L.; Sartorio, R. J. Phys. Chem. B 2001, 105, $328-334$.

(40) Vergara, A.; Paduano, L.; Sartorio, R. Phys Chem. Chem. Phys. 2002, 4, 4716.

(41) Rard, J. A.; Miller, D. G. J. Solution Chem. 1979, 8, 701.

(42) Vergara, A.; Paduano, L.; Vitagliano, V.; Sartorio, R. Macromolecules 2001, 34, 991-1000.

(43) Paduano, L.; Sartorio, R.; D'Errico, G.; Vitagliano, V. J. Chem Soc., Faraday Trans. 1998, 94, 2571.

(44) Castagnolo, D.; Carotenuto, L.; Vergara, A.; Paduano, L.; Sartorio, R. J. Cryst. Growth 2001, 232, 138-148.

(45) Castagnolo, D.; Vergara, A.; Paduano, L.; Sartorio, R.; Annunziata, O. Acta Crystallogr., Sect. D: Biol. Crystallogr. 2002, 58 (10), 16331637. 